\title{
Photo-induced changes in a hybrid amorphous chalcogenide/silica photonic crystal fiber
}

Markos, Christos

Published in:

Applied Physics Letters

Link to article, DOI:

$10.1063 / 1.4861374$

Publication date:

2014

Document Version

Publisher's PDF, also known as Version of record

Link back to DTU Orbit

Citation (APA):

Markos, C. (2014). Photo-induced changes in a hybrid amorphous chalcogenide/silica photonic crystal fiber. Applied Physics Letters, 104(1), [011114 ]. https://doi.org/10.1063/1.4861374

\section{General rights}

Copyright and moral rights for the publications made accessible in the public portal are retained by the authors and/or other copyright owners and it is a condition of accessing publications that users recognise and abide by the legal requirements associated with these rights.

- Users may download and print one copy of any publication from the public portal for the purpose of private study or research.

- You may not further distribute the material or use it for any profit-making activity or commercial gain

- You may freely distribute the URL identifying the publication in the public portal

If you believe that this document breaches copyright please contact us providing details, and we will remove access to the work immediately and investigate your claim. 


\section{AIP Applied Physics \\ Letters}

\section{Photo-induced changes in a hybrid amorphous chalcogenide/silica photonic crystal fiber}

Christos Markos

Citation: Applied Physics Letters 104, 011114 (2014); doi: 10.1063/1.4861374

View online: http://dx.doi.org/10.1063/1.4861374

View Table of Contents: http://scitation.aip.org/content/aip/journal/apl/104/1?ver=pdfcov

Published by the AIP Publishing

\section{Articles you may be interested in}

Retraction: "Photo-induced changes in a hybrid amorphous chalcogenide/silica photonic crystal fiber" [Appl.

Phys. Lett. 104, 011114 (2014)]

Appl. Phys. Lett. 104, 109901 (2014); 10.1063/1.4868137

Extreme optical nonlinearities in chalcogenide glass fibers embedded with metallic and semiconductor nanowires Appl. Phys. Lett. 99, 121102 (2011); 10.1063/1.3641423

Wet-etching selectivity of Ag-photodoped AsGeSeS thin films and the fabrication of a planar corrugated onedimensional photonic crystal by a holographic method

J. Vac. Sci. Technol. B 20, 2017 (2002); 10.1116/1.1505956

Opal photonic crystals infiltrated with chalcogenide glasses

Appl. Phys. Lett. 78, 4094 (2001); 10.1063/1.1380729

Low loss photoinduced waveguides in rapid thermally annealed films of chalcogenide glasses

Appl. Phys. Lett. 74, 13 (1999); 10.1063/1.123118

\section{Asylum Research Atomic Force Microscopes}

Unmatched Performance, Versatility and Support

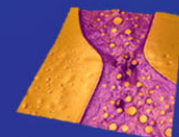

Modulus of Polymers

\& Advanced Materials

Coating Uniformity

\& Roughness

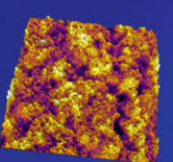

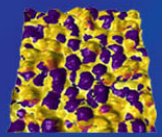

Nanoscale Conductivity

\& Permittivity Mapping
Piezoelectrics

\& Ferroelectrics

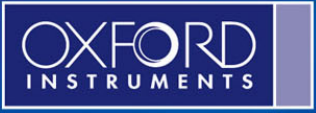

The Business of Science
$+1(805) 696-6466$ sales@AsylumResearch.com www.AsylumResearch.com 


\title{
Photo-induced changes in a hybrid amorphous chalcogenide/silica photonic crystal fiber
}

\author{
Christos Markos ${ }^{\text {a) }}$ \\ DTU Fotonik, Department of Photonics Engineering, Technical University of Denmark, \\ DK-2800 Kgs. Lyngby, Denmark
}

(Received 11 November 2013; accepted 20 December 2013; published online 10 January 2014)

\begin{abstract}
Photostructural changes in a hybrid photonic crystal fiber with chalcogenide nanofilms inside the inner surface of the cladding holes are experimentally demonstrated. The deposition of the amorphous chalcogenide glass films inside the silica capillaries of the fiber was made by infiltrating the nanocolloidal solution-based $\mathrm{As}_{25} \mathrm{~S}_{75}$, while the photoinduced changes were performed by side illuminating the fiber near the bandgap edge of the formed glass nanofilms. The photoinduced effect of the chalcogenide glass directly red-shifts the transmission bandgap position of the fiber as high as $\sim 20.6 \mathrm{~nm}$ at around $1600 \mathrm{~nm}$ wavelength, while the maximum bandgap intensity change at $\sim 1270 \mathrm{~nm}$ was -3 dB. (C) 2014 AIP Publishing LLC. [http://dx.doi.org/10.1063/1.4861374]
\end{abstract}

Photonic crystal fibers (PCFs) also known as microstructured optical fibers have become a major topic of research over the last years. These kinds of fibers are fabricated mainly by a single material (glass or polymer). ${ }^{1-3}$ The crosssection of the fiber consists of a core area surrounded by a periodic array of holes, where the light can be either guided based on index- or bandgap guidance mechanism depending on the refractive index contrast between the core and the cladding. ${ }^{1,4}$ The air-hole cladding structure of PCF enables the infiltration of functional materials and liquids such as liquid crystals, ${ }^{5}$ polymers, ${ }^{6,7}$ etc., into the air holes providing thus the ability to manipulate the guiding properties of the fiber. Consequently, several research groups have already demonstrated all-fiber tunable devices, ${ }^{5}$ filters, ${ }^{6}$ sensors, ${ }^{8}$ etc., using the aforementioned materials. Most of the researched PCFs are oxide based glasses such as fused silica since it is a mature and well-grounded technology. However, oxide based glasses exhibit very limited properties for nonlinear and mid-IR applications.

Chalcogenide glasses, on the other hand, have attracted a lot of scientific interest due to their significant linear and non-linear optical properties such as very high non-linear coefficients, high transparency in the mid-infrared window making them promising candidates for nonlinear optics and IR applications. ${ }^{9,10}$ However, the hallmark property of these glasses is their photosensitivity under illumination with light near their bandgap edge. ${ }^{9}$ In the field of photoinduced effects, the photosensitivity of As-S glasses has been widely studied during the last decades. ${ }^{11-13}$ The observed changes in optical, electrical, mechanical, and rheological properties have been always linked to changes in atomic arrangements within the glass structure. ${ }^{12}$ However, the photochemical and electrochemical processes are still not well understood in this class of amorphous materials.

Direct fabrication of high-quality chalcogenide PCFs is a complex and difficult task since chalcogenide glasses exhibit steep viscosity-temperature profile and low mechanical stability compared to silica. ${ }^{14}$ Therefore, an efficient route

\footnotetext{
${ }^{\text {a) }}$ Author to whom correspondence should be addressed. Electronic mail: chmar@fotonik.dtu.dk. Tel.: +45 452536 79. Fax: +45 45936581.
}

would be to combine the mature silica PCFs with chalcogenide glasses and benefit from the advantages of both materials. Limited research has been done so far on the infiltration of PCF with chalcogenide glasses. Recently, Granzow et $a l .{ }^{15}$ reported the infiltration of a sulfide-based chalcogenide glass into the holes of a PCF using the pressure-assisted melt-filling technique. However, the proposed method requires a highly specialized and costly custom-made equipment since the glass has to be molten at high temperature (i.e., $665^{\circ} \mathrm{C}$ ) and then apply high pressure to fill the air holes of the PCF a few millimeters.

In this work, we report photoinduced effects occurring to the chalcogenide glass deposited inside the holes of a PCF. We show the possibility to modify the guiding properties of a chalcogenide-filled PCF by using an external low power continuous-wave (CW) laser source near the bandgap of the infused chalcogenide glass. The integration of the chalcogenide glasses into the silica PCF was made in a simple and rather inexpensive way compared to melt-filling technique, simply by using a nanocolloidal solution-based As-S glass. ${ }^{16}$ The solution was infiltrated into the holes of the PCF and by annealing the fiber at low temperature, the remaining solvent removed, forming thus a nanometer-thick layer of the desired chalcogenide glass in the inner surface of the PCF's air-holes. The solution-based chalcogenide glasses offer a great flexibility and several advantages for the development of hybrid PCFs with most important ones to be the simplicity of combining any desired chalcogenide material with silica PCF at ambient conditions and the possibility of multi-layer deposition of different glass compositions, which is presently not possible with any other fabrication technique. We present experimentally how the photoinduced structural effects introduced to the hybrid fiber using a diode laser at $473 \mathrm{~nm}$ (near the bandgap edge of $\mathrm{As}_{25} \mathrm{~S}_{75}$ ) can relocate the transmission bandgap position of the fiber. The structural rearrangements of the formed $\mathrm{As}_{25} \mathrm{~S}_{75}$ nanolayers inside PCF due to the high absorption of light at frequency near the absorption band of the material lead to changes in the properties of the glass such as the refractive and density. ${ }^{12}$ Consequently, a small change to the refractive index and/or density can directly affect the position of the bandgap. 
The preparation of the nanocolloidal solution-based $\mathrm{As}_{25} \mathrm{~S}_{75}$ was made by mixing high purity As and $\mathrm{S}$ elements in a quartz ampoule. The ampoule with the elements was heated to $\sim 700^{\circ} \mathrm{C}$ and then the melt was quenched to room temperature. The bulk glass was dissolved in n-butylamine (Alfa Aesar, 99.5\%) and the dissolution process was carried out inside a sealed glass container to prevent solvent evaporation. A hot plate with magnetic stirrer was used to expedite the dissolution process. The concentration of the final solution was $0.2 \mathrm{~g} / \mathrm{ml}$ and the viscosity of the solution was less than $0.25 \mathrm{cP} .{ }^{17}$ The dissolution process took several days in order to ensure complete homogenization. The resulting solution was stored inside a nitrogen-purged glove box until use to prevent oxygen and water contamination.

For our experiments, we employed a commercially available PCF purchased by NKT photonics A/S. The cladding hole diameter of the fiber is $\mathrm{d}=3.5 \mu \mathrm{m}$, while the pitch $\Lambda$ (distance between the holes) is $7.7 \mu \mathrm{m}$. The core of the fiber is $\sim 11 \mu \mathrm{m}$. The scanning electron microscopy of the cross-section of the fiber is shown in Fig. 1(a). The nanocolloidal solution was infiltrated inside the holes of the fiber using capillary effects where for an infused length of around $5 \mathrm{~cm}$, the infiltration time was $\sim 10 \mathrm{~min}$. It is worth noting that the infiltrated fiber length is directly related with the viscosity of the material, the size of the holes, and the applied pressure to the sample as it has been extensively described before. ${ }^{18}$ After the infiltration of the fiber, the sample was rested inside a fume hood at room temperature for about 2 days in order to allow smoothly the solvent evaporation. We further annealed the fiber at $60^{\circ} \mathrm{C}$ for $6 \mathrm{~h}$ to remove any solvent residues. After the annealing of the fiber, the final chalcogenide film was formed inside the silica capillaries in a uniform and smooth manner. Figure 1(b) shows the SEM image of the final deposited chalcogenide films around the core of fiber, while Fig. 1(c) shows a magnified SEM image, which clearly indicates the existence of the chalcogenide film to the surface of the hollow channel of the fiber. The thickness of the chalcogenide film was not possible to be
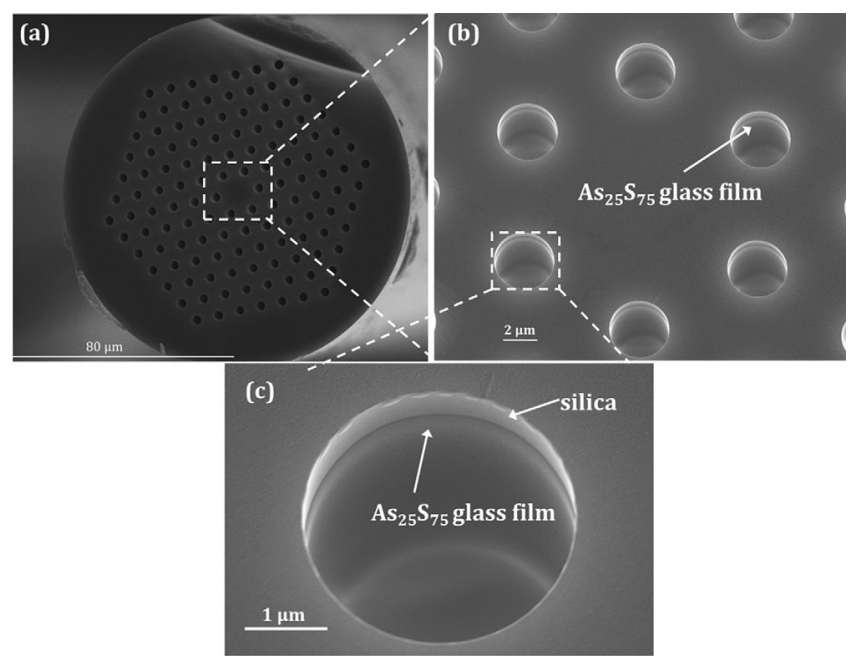

FIG. 1. (a) SEM image of the cross-section of the fiber. (b) SEM image around the core indicated the formed thin chalcogenide films inside the silica capillaries of the fiber and (c) magnified SEM image of a single hole shown clearly the nanometer thick chalcogenide film deposited on the surface of the hole. determined accurately since it was less than $10 \mathrm{~nm}$. The refractive index of the nanolayers is around $2.1-2.5{ }^{19}$ however, both the annealing time and temperature have a crucial role to the final refractive index of the glass layers as reported previously. ${ }^{19}$

In order to further confirm the existence of the chalcogenide films inside the holes of the PCF, we used Energydispersive X-ray (EDX) spectroscopy to identify the elements of the formed film. Figure 2(a) shows the SEM image of an angle-cleaved hole with chalcogenide film and the point where the EDX was applied. In order to identify the elements of the thin film, the acquisition time of the EDX measurement was more than 10 min. As it can be seen from Fig. 2(b), the EDX spectrum indicates the existence of arsenic (As) and sulfide (S) elements to the nanofilms, which further confirms the structural existence of the formed glass layer. In order to identify the absorbance edge of the material, we drop casted the nanocolloidal glass solution to a microscope slide. The measurement of the absorbance spectrum was performed using an FTNIR spectrophotometer (Bruker Vector). Since the composition of the glass is a sulfur rich-based chalcogenide, the bandgap absorption edge has been shifted to shorter wavelengths compared, for example, to stoichiometric $\mathrm{As}_{2} \mathrm{~S}_{3} .{ }^{16}$ Figure 2(c) shows the absorbance spectrum of the $\mathrm{As}_{25} \mathrm{~S}_{75}$ chalcogenide think film for a range from $400 \mathrm{~nm}$ to $1800 \mathrm{~nm}$ indicating that the photostructural effects can be revealed using a diode laser at $473 \mathrm{~nm}$, which is close enough to the bandgap edge of the material.

For the optical characterization of the hybrid chalcogenide/silica PCF, a supercontinuum laser source was used covering from 500 to $1750 \mathrm{~nm}$ wavelength. The output beam was collected with a microscope objective and a multimode fiber, while the transmitted signal was monitored on an optical spectrum analyzer (OSA). The cladding light was blocked by inserting an iris diaphragm into the beam path, such that only light from the fiber core was recorded. The illumination of the sample near the bandgap edge was made using a $50 \mathrm{~mW} \mathrm{CW}$ laser source at $473 \mathrm{~nm}$. The fiber sample
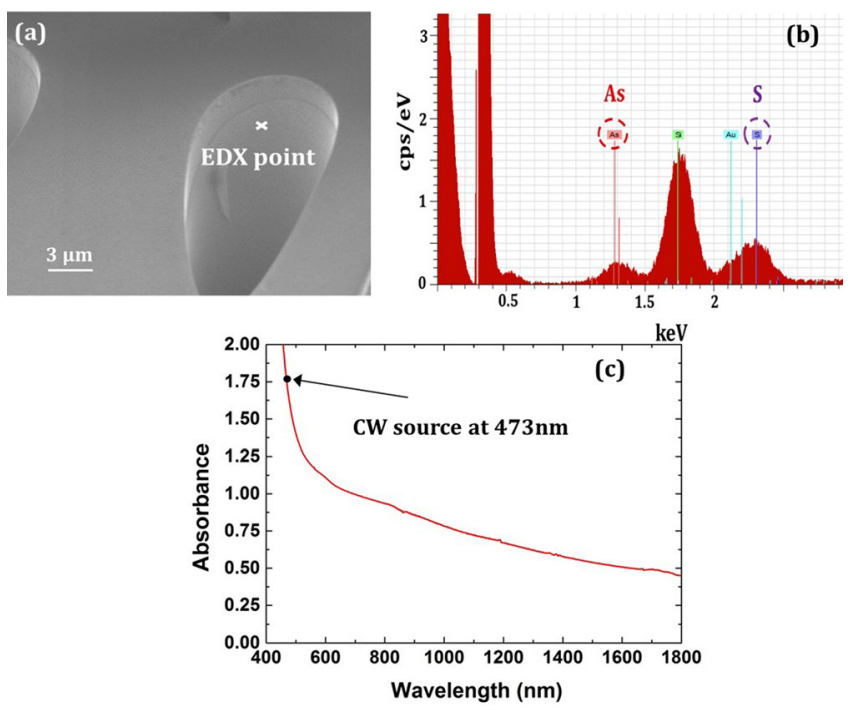

FIG. 2. (a) SEM image of an angled-cleave section of the fiber indicated the EDX point. (b) EDX spectrum of the chalcogenide film showing the existence of As-S elements. (c) Absorbance spectrum of $\mathrm{As}_{25} \mathrm{~S}_{75}$ starting from 400 up to $1800 \mathrm{~nm}$ wavelength. 
was also laser scanned forward and backward using a linear translation drive stage (Thorlabs, DDSM100), while the beam of the laser was focused on the sample using an objective lens $(\times 10)$. The near field pattern of the fundamental guiding mode was recorded using a beam splitter and a CCD camera. The experimental set-up is shown in Fig. 3(a).

The guiding mechanism in a conventional PCF is based on total internal reflection (TIR). By infiltrating the holes of the fiber with a high index material (chalcogenide glass layers in this case), the average refractive index of the cladding is consequently higher than the silica core. The guiding mechanism is then modified to ARROW (antiresonant reflecting optical waveguide) guidance. In ARROW guidance, the high index materials consisting the effective cladding of the fiber are considered as separate waveguides where each waveguide can support a number of guided modes. If light impinging on the inclusion is on transverse resonance with one of its guided modes, then it can propagate through the inclusion and escape from the core. ${ }^{20}$ Similarly, if light is in an off-resonance with the modes in the high index materials then it is reflected and confined back to the core of the fiber. Consequently, the transmission spectrum exhibits several minimum and maximum bands. ${ }^{20}$ Figure 3(b) shows the overexposed near field pattern of the fundamental mode recorded using a supercontinuum generation. The distortion to the near field pattern of the mode was introduced by the cleaved end facet of the fiber. Nevertheless, it can be seen that the amount of light is guided on the high index chalcogenide films deposited to the holes of the fiber cladding. Figure 3(c) shows the normalized transmission spectrum of the chalcogenide-filled PCF
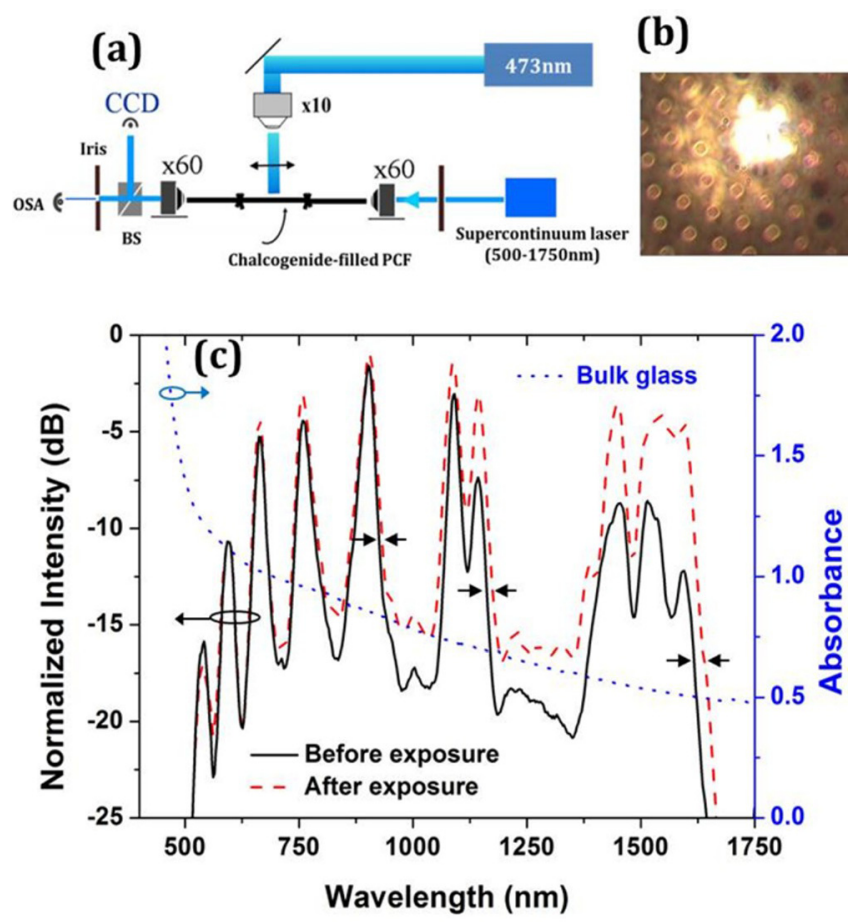

FIG. 3. (a) Experimental set-up. (b) Overexposed near-field pattern of the fundamental guiding mode. (c) Transmission spectrum before (black-solid line) and after (red-dashed line) illumination of the hybrid chalcogenide PCF with near bandgap light at $473 \mathrm{~nm}$. The blue dotted line corresponds to the absorbance spectrum of the bulk chalcogenide glass. (black line). The high index layers in the cladding of the fiber strongly reveal full photonic bandgaps from 500 to $1700 \mathrm{~nm}$ wavelength. For the short wavelengths, there are 5 district narrow bands with the maximum bandwidth of the guiding band to be around $46.5 \mathrm{~nm}$ at around $900 \mathrm{~nm}$ wavelength, while for wavelengths longer than $1000 \mathrm{~nm}$, the bandgap bandwidth significantly increases with maximum bandwidth at $1520 \mathrm{~nm}$ wavelength to be around $122 \mathrm{~nm}$. Figure 3(c) also shows the red-shifting of the location of the bandgaps after the illumination of the sample with $\mathrm{CW}$ laser source at $473 \mathrm{~nm}$ wavelength (red dotted line) at maximum power (50 $\mathrm{mW}$ ). The laser beam scanned the sample at a constant speed of $80 \mathrm{~mm} / \mathrm{min}$ in order to ensure complete photostructural homogeneity along the length of the filled fiber. The sample was scanned 3 times in total (forward and reverse), with a total illumination time of $\sim 18 \mathrm{~min}$, while further bandgap light illumination of the fiber did not enhance the photoinduced changes. The final delivered power at the high index layers of the air-holes close to the core is less than $50 \mathrm{~mW}$ since the amount of the power is side scattered by the continuous air-silica interface along the penetration path.

When chalcogenide glasses are exposed to the appropriate light, several distinct photo-induced structural and physiochemical changes have been observed. Some of them are photo-polymerization, photo-crystallization, photodecomposition (in compounds), photo-induced morphological changes, and light-induced changes in local atomic configuration. Among the aforementioned photoinduced effects in these glasses, the most notable one is photodarkening. This effect is believed to originate from the decomposition of the arsenic-chalcogen bond and the formation of the defects in the glassy structure (arsenic clusters). ${ }^{11}$ In this case, the illumination of the sample with light near bandgap edge of the deposited chalcogenide films causes a change to the refractive index of the formed glass films. This is based on the fact that the refractive index and the absorption coefficient of the material, respectively, are not independent from each other and are related through the Kramers-Kronig relationship. ${ }^{21}$ Since the bandgap light illumination of chalcogenide glass introduces photodarkening effects to the formed glass, the refractive index will thus change as high as $\Delta \mathrm{n} \approx 0.07$ in films ${ }^{22}$ and as $\Delta \mathrm{n} \approx 10^{-3}$ in fibers. ${ }^{23}$ Figure 4(a) shows the bandgap right edge red-shift of the transmission spectrum after illumination (Fig. 3(c), red line) by tracking each edge separately. It is shown clearly that the photostructural effect is dominant at longer wavelengths with maximum bandgap red-shifting of $20.69 \mathrm{~nm}$ at $1633 \mathrm{~m}$ wavelength. The photoinduced change affects also the bandgap intensity where as it can been seen from Figure 4(b) the reduction of the bandgap varies monotonically with respect to the corresponding wavelength. The maximum reduction to the bandgap intensity was measured to be $-3 \mathrm{~dB}$ at around $1270 \mathrm{~nm}$.

In conclusion, photoinduced effects occurring in a hybrid chalcogenide/silica PCF under the side illumination with the near bandgap light are experimentally demonstrated. The nanocolloidal solution-based chalcogenide glass integrated inside the cladding holes of a commercially available $\mathrm{PCF}$, whereas the near bandgap laser illumination of the sample at $473 \mathrm{~nm}$ introduced a direct red-shift to the 

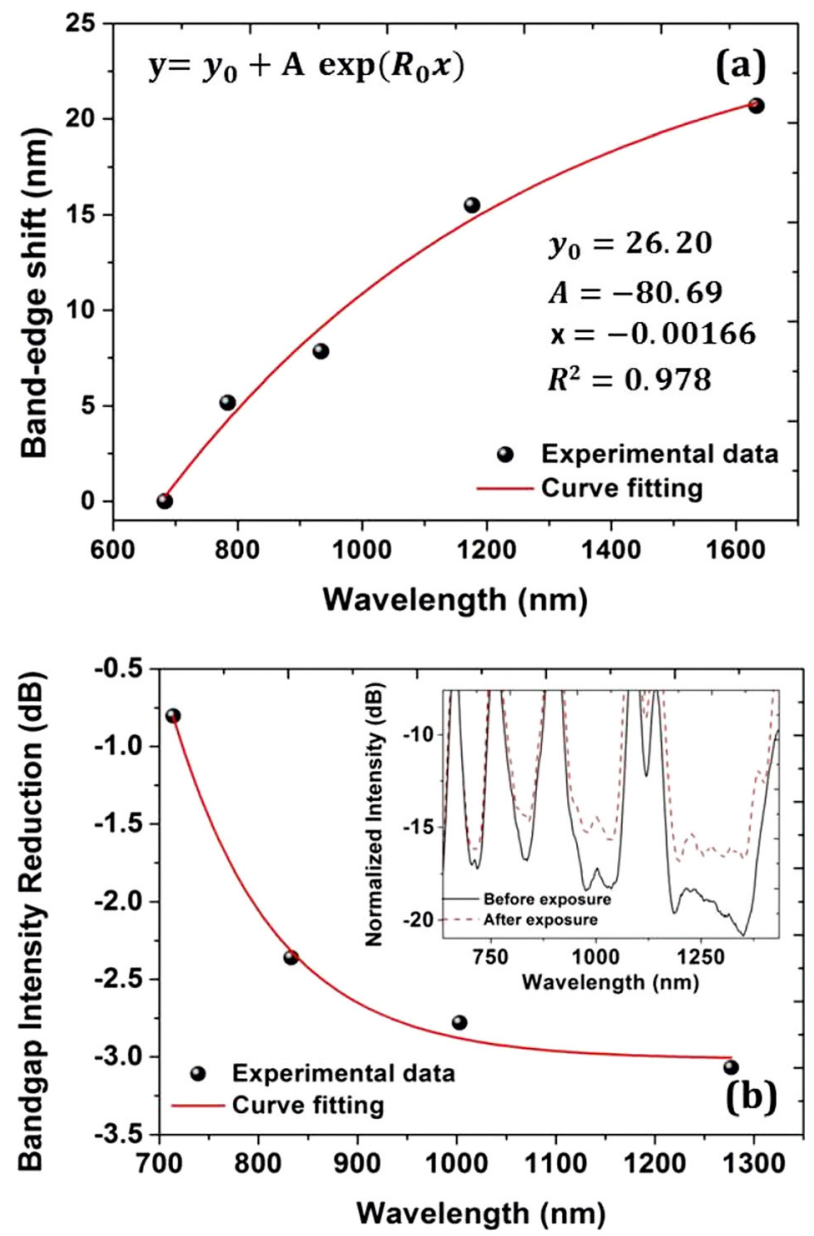

FIG. 4. (a) Bandgap edge shift vs the wavelength of each transmission band. (b) Intensity reduction of each bandgap vs the corresponding wavelength. Inset: Demonstration of the fiber bandgaps tracked.

transmission bandgap spectrum as high as $20.6 \mathrm{~nm}$ at 1633 , while the bandgap intensity reduced around $-3.1 \mathrm{~dB}$ at $1270 \mathrm{~nm}$ wavelength. Further optimization of the performance of the fiber device can be achieved by using higher laser energies or by further enhancement of the chalcogenide glass's photosensitivity. Other previous reports have demonstrated light guidance in the mid-IR transmission window by combining chalcogenide and silica glass in a step-index fiber form where infiltration of PCFs with soft-glasses has been achieved by using the melt-filling technique. ${ }^{24,25}$ However, the main scope of this work is to demonstrate the ability of combining silica-based PCF with chalcogenide glasses in a simple way for the development of all-fiber tunable bandgap devices. Furthermore, the proposed method for fabricating hybrid PCFs combined with the unique optical properties of chalcogenide glasses opens a new route for all-fiber filters at visible range as well as tunable devices.

C.M. would like to acknowledge the Hans Christian Ørsted post-doc program for the financial support.

${ }^{1}$ T. A. Birks, J. C. Knight, and P. St. J. Russell, Opt. Lett. 22, 961 (1997).

${ }^{2}$ M. A. van Eijkelenborg, M. C. J. Large, A. Argyros, J. Zagari, S. Manos, N. A. Issa, I. Bassett, S. Fleming, R. C. McPhedran, C. M. de Sterke, and N. A. P. Nicorovici, Opt. Express 9, 319 (2001).

${ }^{3}$ C. Markos, A. Stefani, K. Nielsen, H. K. Rasmussen, W. Yuan, and O. Bang, Opt. Express 21, 4758 (2013).

${ }^{4}$ P. Domachuk, H. C. Nguyen, B. J. Eggleton, M. Straub, and M. Gu, Appl. Phys. Lett. 84, 1838 (2004).

${ }^{5}$ L. Wei, T. T. Alkeskjold, and A. Bjarklev, Appl. Phys. Lett. 96, 241104 (2010).

${ }^{6} \mathrm{G}$. Zito and S. Pissadakis, Opt. Lett. 38, 3253 (2013).

${ }^{7}$ C. Markos, K. Vlachos, and G. Kakarantzas, Opt. Mater. Express 2, 929 (2012).

${ }^{8}$ W. Yuan, L. Wei, T. T. Alkeskjold, A. Bjarklev, and O. Bang, Opt. Express 17, 19356 (2009).

${ }^{9}$ B. J. Eggleton, B. Luther-Davies, and K. Richardson, Nat. Photonics 5, 141 (2011).

${ }^{10}$ J. S. Sanghera and I. D. Aggarwal, J. Non-Cryst. Solids 256, 6 (1999).

${ }^{11}$ C. Y. Yang, M. A. Paesler, and D. E. Sayers, Phys. Rev. B: Condens. Matter 36, 9160 (1987).

${ }^{12}$ A. B. Seddon, J. Non-Cryst. Solids 184, 44-50 (1995).

${ }^{13}$ S. N. Yannopoulos, Phys. Rev. B 68, 064206 (2003).

${ }^{14}$ L. Brilland, F. Smektala, G. Renversez, T. Chartier, J. Troles, T. Nguyen, N. Traynor, and A. Monteville, Opt. Express 14, 1280 (2006).

${ }^{15}$ N. Granzow, P. Uebel, M. A. Schmidt, A. S. Tverjanovich, L. Wondraczek, and P. St. J. Russell, Opt. Lett. 36, 2432 (2011).

${ }^{16}$ C. Markos, S. N. Yannopoulos, and K. Vlachos, Opt. Express 20, 14814 (2012).

${ }^{17}$ T. A. Guiton and C. G. Pantano, Chem. Mater. 1, 558 (1989).

${ }^{18}$ K. Nielsen, D. Noordegraaf, T. Sørensen, A. Bjarklev, and T. P. Hansen, J. Opt. A: Pure Appl. Opt. 7, L13 (2005).

${ }^{19}$ S. Song, J. Dua, and C. B. Arnold, Opt. Express 18, 5472 (2010).

${ }^{20}$ N. M. Litchinitser, A. K. Abeeluck, C. Headley, and B. J. Eggleton, Opt. Lett. 27, 1592 (2002).

${ }^{21}$ L. Calvez, Z. Yang, and P. Lucas, J. Phys. D: Appl. Phys. 43, 445401 (2010).

${ }^{22}$ E. Zou, B. X. Chen, H. S. Lin, H. Hamanaka, and M. Iso, Appl. Opt. 48, 6442 (2009).

${ }^{23}$ F. Luan, E. Magi, T. Gong, I. Kabakova, and B. J. Eggleton, Opt. Lett. 36, 4761 (2011).

${ }^{24}$ N. Granzow, M. A. Schmidt, W. Chang, L. Wang, Q. Coulombier, J. Troles, P. Toupin, I. Hartl, K. F. Lee, M. E. Fermann, L. Wondraczek, and P. St. J. Russell, Opt. Express 21, 10969 (2013).

${ }^{25}$ N. Da, L. Wondraczek, M. A. Schmidt, N. Granzow, and P. St. J. Russell, J. Non-Cryst. Solids 356, 1829 (2010). 